Chapman University

Chapman University Digital Commons

Mathematics, Physics, and Computer Science

Science and Technology Faculty Articles and

Faculty Articles and Research

Research

1980

\title{
The Evolution of Supernova Remnants in Different Galactic Environments, and its Effects on Supernova Statistics
}

Menas Kafatos

Chapman University, kafatos@chapman.edu

S. Sofia

Univ Estadual Londrina

C. Bruhweilier

Catholic University of America

T. R. Gull

NASA, Goddard Space Flight Center

Follow this and additional works at: http://digitalcommons.chapman.edu/scs_articles

Part of the Stars, Interstellar Medium and the Galaxy Commons

\section{Recommended Citation}

Kafatos, M., Sofia, S., Bruhweiler, F.C., Gull, T.R. (1980) The Evolution of Supernova Remnants in Different Galactic Environments, and its Effects on Supernova Statistics, Astrophysical Journal, 242: 294-305. doi: 10.1086/158463

This Article is brought to you for free and open access by the Science and Technology Faculty Articles and Research at Chapman University Digital Commons. It has been accepted for inclusion in Mathematics, Physics, and Computer Science Faculty Articles and Research by an authorized administrator of Chapman University Digital Commons. For more information, please contact laughtin@chapman.edu. 


\section{The Evolution of Supernova Remnants in Different Galactic Environments, and its Effects on Supernova Statistics}

\section{Comments}

This article was originally published in Astrophysical Journal, volume 242, in 1980. DOI: 10.1086/158463

\section{Copyright}

IOP Publishing 


\title{
THE EVOLUTION OF SUPERNOVA REMNANTS IN DIFFERENT GALACTIC ENVIRONMENTS, AND ITS EFFECTS ON SUPERNOVA STATISTICS
}

\author{
Minas Kafatos, ${ }^{1}$ Sabatino Sofia, ${ }^{2}$ Frederick Bruhweiler, ${ }^{3}$ and Theodore Gull ${ }^{2}$ \\ Received 1980 February 5; accepted 1980 May 21
}

\begin{abstract}
By examining the interaction between supernova (SN) ejecta and the various environments in which the explosive event might occur, we conclude that only a small fraction of the many SNs produce observable supernova remnants (SNRs). This fraction, which is found to depend weakly upon the lower mass limit of the SN progenitors, and more strongly on the specific characteristics of the associated interstellar medium, decreases from approximately $15 \%$ near the galactic center to $10 \%$ at $R_{\text {gal }} \sim 10 \mathrm{kpc}$ and drops nearly to zero for $R_{\text {gal }}>15 \mathrm{kpc}$. Generally, whether a SNR is detectable is determined by the density of the ambient interstellar medium in which it is embedded. We find that SNRs are only detectable above some critical density $\left(n \sim 0.1 \mathrm{~cm}^{-3}\right)$. The presence of large, low-density superbubble cavities around stellar associations due to the combined effects of stellar winds and supernova shells strongly suggests that a large portion of the detectable SNRs must have runaway stars as their progenitors. These results explain the differences between the substantially larger SN rates in the Galaxy derived both from pulsar statistics and from observations of SN events in external galaxies, when compared to the substantially smaller SN rates derived from galactic SNR statistics. These results also explain the very large number of SNRs observed toward the galactic center in comparison to few SNRs found in the anticenter direction.
\end{abstract}

Subject headings: nebulae: supernova remnants - stars; stellar statistics

\section{INTRODUCTION}

The evolution of a SNR expanding within the "typical" interstellar medium (i.e., number density $n \sim 1$ $\mathrm{cm}^{-3}$, temperature $\left.T \sim 10^{2}-10^{4} \mathrm{~K}\right)$ has been studied with a great deal of detail and sophistication (for a recent review see Chevalier 1977). In particular, detailed integrations of the basic shock equations have been performed (Chevalier 1974; Mansfield and Salpeter 1974), and the complex phenomena which appear during the transition from the adiabatic to the isothermal phase have been addressed (Chevalier 1975; Chevalier and Theys 1975; Woodward 1976; McCray, Stein, and Kafatos 1975, etc.).

Recognizing the inhomogeneous nature of the interstellar medium (ISM), considerable work has been carried out to study the effects of inhomogeneities in the structure and evolution of the SN shock waves. For example, McKee and Cowie (1975), Sgro (1975), and Woodward (1976) have investigated the interaction of supernova shock waves with interstellar clouds, and McKee and Ostriker (1977) have examined the effects of SN explosions on a cloudy interstellar medium.

\footnotetext{
${ }^{1}$ George Mason University, Physics Department.

${ }^{2}$ Goddard Space Flight Center, Greenbelt, MD.

${ }^{3}$ Computer Sciences Corporation, Silver Spring, MD.
}

In all the above work, the basic shock is always assumed to propagate within a typical (as defined earlier) interstellar medium, and until quite recently no attempt had been made to study the evolution of a SN shock wave expanding into media representing the various possible environments of supernova progenitors within the Galaxy. For example, it is well known that most (if not all) stars are born in groups (clusters or associations). The precursor of the stellar association is a dense molecular cloud. The first supernova from the stars in the group is set off near, perhaps inside, dense, cold gaseous media. The evolution and long term detectability of such an event (recently studied by Wheeler, Mazurek, and Sivaramakrishnan 1980; Shull 1980 ) is obviously very different from that of the canonical supernova remnant that is propagating in the typical interstellar medium.

Supernovae propagating in dense molecular clouds will be very rare, however, since the first one may disrupt one of the smaller clouds (Wheeler, Mazurek, and Sivaramakrishnan 1980), whereas for the more massive clouds the combined effect of stellar winds and the earliest supernovae (Bruhweiler et al. 1980) creates an expanding, hot, low-density cavity, within which subsequent supernova shells will expand for times up to tens of thousands of years. It is this scenario, in fact, 
which is by far the most common encountered by supernova shells. As we shall discuss in $\S \mathrm{V}$, however, since most massive stars occur in binary systems, when the primary member of the system becomes supernova, it may impart a large velocity to the secondary star by means of the slingshot effect (Blaauw 1964). Many of these stars will in fact overtake the supershell and remain within the confines of the galactic disk until they become supernovae. These stars are the ones that produce the typical supernova remnants so extensively discussed in the scientific literature.

In this paper, we will study the evolution of supernova shells into these very dissimilar media. Because of the dramatic effects of the presupernova environment on the evolution of the remnant, we have confined our theoretical discussion on the simplest possible description. Thus, following Spitzer (1978), we characterize the supernova shock by three phases, namely, $(a)$ initial free expansion of the supernova material, $(b)$ intermediate adiabatic (or Sedov) expansion, and (c) late isothermal expansion. Although the structure of the presupernova environment can, in general, be fairly complex, it is usually made up of a combination of dense clouds, a diffuse, warm medium, and rarefied, hot cavities. To that extent, we shall study the evolution of supernova remnants into these three types of media, which should span conditions for nearly all real cases. Thus, we will consider the remnant evolution into (1) a dense $\left(n \sim 10^{4} \mathrm{~cm}^{-3}\right)$, cold $\left(T \lesssim 10^{2} \mathrm{~K}\right)$ molecular cloud, (2) the typical interstellar medium $(n \sim 1$ $\left.\mathrm{cm}^{-3}, T \sim 10^{2}-10^{4} \mathrm{~K}\right)$, and (3) a bubble or supernova cavity $\left(n \sim 10^{-2} \mathrm{~cm}^{-3}, T \sim 5 \times 10^{5} \mathrm{~K}\right)$.

\section{TYPES OF SUPERNOVA REMNANTS}

Before describing each particular type of SNR, let us introduce the relations which allow us to compute the several SN phases for each particular environment. As stated earlier, we shall follow the formalism of Spitzer (1978) and Gorenstein and Tucker (1976).

In the early phase, $(a)$, a shock wave will travel just ahead of the ejected shell, with a velocity $V_{S}$. The shock will heat matter to a temperature

$$
T_{S}=3 \mu m_{\mathrm{H}} V_{S}^{2} / 16 k \approx 1.3 \times 10^{-9} V_{S}^{2}
$$

(Landau and Lifshitz 1959), where $m_{\mathbf{H}}$ is the mass of the hydrogen atom, $\mu$ is the mean molecular weight, and $k$ is the Boltzmann constant. This phase ends when the swept-up interstellar material equals the mass of the ejecta, i.e., when

$$
R_{S}=\left(\frac{3 M_{\mathrm{ej}}}{4 \pi \rho}\right)^{1 / 3}
$$

where $M_{\mathrm{ej}}$ is the mass of the ejecta and $\rho$ the density of the ambient interstellar medium. The elapsed time $t$ between the supernova event and the end of phase (a) is given by

$$
t=R_{S} / V_{S}
$$

Phase $(b)$, the intermediate nonradiative expansion phase, can be computed by means of the Sedov solution. The temperature immediately behind the shock is given by

$$
T_{2}=\frac{3 \mu m_{\mathrm{H}} V_{S}^{2}}{16 k}=\frac{0.061 \mu}{k} \frac{E}{\rho R_{S}^{3}} \mathrm{~K},
$$

where $E$ is the kinetic energy of the ejecta,

$$
R_{S}=(2.02 E / \rho)^{1 / 5} t^{2 / 5} \mathrm{~cm}
$$

and

$$
V_{S}=\left(\frac{3.04 E}{3 \pi \rho}\right)^{1 / 2}\left(\frac{1}{R_{S}}\right)^{3 / 2} \mathrm{~cm} \mathrm{~s}^{-1}
$$

and $t$ can again be obtained from (3).

Phase $(b)$ ends when $T$ falls below $\sim 10^{6} \mathrm{~K}$, for $n \sim 1$ $\mathrm{cm}^{-3}$, since radiative cooling then becomes important, which brings the onset of phase $(c)$, the late isothermal expansion. This phase can be represented by the snowplow model, where conservation of momentum applies. Here the shell velocity is given by

$$
V_{S}=\frac{3}{4} \frac{M_{1} V_{1}}{\pi \rho}\left(\frac{1}{R_{S}}\right)^{3},
$$

where $M_{1}$ and $V_{1}$ are, respectively, the shell mass and velocity at the end of phase $(b)$. At this phase, most of the swept-up material is in a cool, dense shell, although there may be some thermal X-radiation from the hot low-density gas interior to the shell. This low-density interior gas has a long cooling time.

We now discuss the three example SNRs and how differently they appear in the several expansion phases. In all of our calculations we shall assume that, during the $\mathrm{SN}$ event, $4 M_{\odot}$ of the stellar material are ejected with a velocity of $5000 \mathrm{~km} \mathrm{sec}^{-1}$, and thus, the total kinetic energy of the ejecta is $10^{51}$ ergs. These parameters are a realistic representation of a Type II SN which would be expected to occur in an $\mathrm{OB}$ association.

\section{a) A Supernova in a Dense Molecular Cloud}

While molecular clouds found in nature have a large range of size, mass, and density, a typical molecular cloud can be approximated as a sphere with uniform density $n_{\mathrm{H}_{2}} \sim 10^{4} \mathrm{~cm}^{-3}$ having a diameter $D_{C} \sim 5 \mathrm{pc}$ (i.e., a cloud mass $M_{C} \sim 3.5 \times 10^{4} M_{\odot}$ ), and with a temperature $T<10^{2}$ (Burton 1976).

Wheeler, Mazurek, and Sivaramakrishnan (1980) and Shull (1980) have modeled the effects of a SN explod- 
TABLE 1A

The Molecular Cloud SNR $\left(n=10^{4} \mathrm{~cm}^{-3}, T \leqq 10^{2} \mathrm{~K}\right)$

\begin{tabular}{rrrll}
\hline \hline \multicolumn{1}{l}{$t(\mathrm{yr})$} & $V_{S}\left(\mathrm{~km} \mathrm{~s}^{-1}\right)$ & $T_{S}(\mathrm{~K})$ & $R_{S}(\mathrm{pc})$ & \multicolumn{1}{c}{ Remarks } \\
\hline $0 \ldots \ldots \ldots$ & 5000 & $3.5 \times 10^{8}$ & 0 & Explosion occurs \\
$23 \ldots \ldots \ldots$ & 5000 & $3.5 \times 10^{8}$ & 0.12 & Free expansion ends \\
$112 \ldots \ldots \ldots$ & 1200 & $2 \times 10^{7}$ & 0.3 & Adiabatic phase ends \\
$9 \times 10^{4} \ldots$ & 5 & $2.5 \times 10^{3}$ & 1.9 & Shell stalls \\
\hline
\end{tabular}

TABLE 1B

The Hot CAVITY SNR $\left(n \sim 10^{-2} \mathrm{~cm}^{-3}, T \sim 5 \times 10^{5} \mathrm{~K}\right)$

\begin{tabular}{|c|c|c|c|c|}
\hline$t(\mathrm{yr})$ & $V_{S}\left(\mathrm{~km} \mathrm{~s}^{-1}\right)$ & $T_{S}(\mathbf{K})$ & $R_{S}(\mathrm{pc})$ & Remarks \\
\hline $0, \ldots \ldots$ & 5000 & $3.5 \times 10^{8}$ & 0 & Explosion occurs \\
\hline $3.7 \times 10^{3}$. & 5000 & $3.5 \times 10^{8}$ & 19 & Free expansion ends \\
\hline $1.4 \times 10^{5} \ldots$ & 300 & $1.3 \times 10^{6}$ & 105 & $\begin{array}{l}\text { SN shock encounters moving bubble } \\
\text { shell, and it quickly gets decelerated to } \\
\text { the bubble velocity }\left(21 \mathrm{~km} \mathrm{~s}^{-1}\right)\end{array}$ \\
\hline
\end{tabular}

TABLE IC

The Classical SNR $\left(n \sim 1 \mathrm{~cm}^{-3}, T \sim 10^{2}-10^{4} \mathrm{~K}\right)$

\begin{tabular}{lrcrl}
\hline \hline$t(\mathrm{yr})$ & $V_{S}\left(\mathrm{~km} \mathrm{~s}^{-1}\right)$ & $T_{S}(\mathrm{~K})$ & $R_{S}(\mathrm{pc})$ & \multicolumn{1}{c}{ Remarks } \\
\hline $0 \ldots \ldots \ldots \ldots$ & 5000 & $3.5 \times 10^{8}$ & 0 & Explosion occurs \\
$6.2 \times 10^{2} \ldots$ & 5000 & $3.5 \times 10^{8}$ & 3.1 & Free expansion ends \\
$2.9 \times 10^{4} \ldots$ & 265 & $10^{6}$ & 19.6 & Adiabatic phase ends \\
$2.6 \times 10^{6} \ldots$ & 5 & $2.5 \times 10^{3}$ & 73.6 & Shell stalls \\
\hline
\end{tabular}

ing within a molecular cloud. The characteristics of the SNR at the end of each phase using their results are summarized in Table 1 . We will subsequently call these SNRs the molecular cloud SNRs.

Because of the very high density, the phases occur rapidly for the molecular cloud SNRs. Wheeler, Mazurek, and Sivaramakrishnan (1980) indeed suggest that the adiabatic phase may not exist if $n_{\mathrm{H}_{2}}>10^{5}$ $\mathrm{cm}^{-3}$. Whether this happens depends on $n$ as well as on $M_{\mathrm{ej}}$. The molecular cloud SNR represents the conditions within a young molecular cloud when the first, most massive stars become supernovae. These conditions would be very short-lived as the SNRs would push the molecular cloud away from the remaining massive stars within the association and cause rapid cloud fragmentation (Elmegreen 1979). Consequently, this picture will apply to a relatively small minority of the SNs which occur in the Galaxy. Moreover, at no point of its evolution does the resulting shell remotely resemble the familiar observed supernova remnant (SNR). In particular, during the earlier stages of evolution, optical and X-ray observations are useless as a means of detection because the cloud is optically thick to those wavelengths. If the $\mathrm{SN}$ were to produce a $\gamma$-ray pulsar as the stellar remnant, it would be observable. However, since only a minority of the pulsars are known to emit $\gamma$-rays, this is not an effective means to search for SNs within dense clouds. The SNR should be detectable by means of the infrared emission from the heated grains inside and outside of the cloud (Wheeler, Mazurek, and Sivaramakrishnan 1980; Shull 1980; Silk and Burke 1974). Even this technique is not foolproof, though, since it may be very difficult to differentiate the cloud-embedded source of infrared emission as a SNR rather than a recently formed OB association.

\section{b) A Supernova within a Hot, Rarefied Cavity}

Most supernovae which occur in the older OB associations will first expand into the hot highly evacuated volume produced by the combined effects of stellar winds and earlier SNs (Bruhweiler et al. 1980). The ejecta expand freely until enough gas is encountered to form a shock. The ejecta do not encounter significant gas until very late in the evolution of the SNR. Indeed, for a few thousands of years the SNR is hot, dilute gas expanding without bound. Such a gas is very difficult to observe (the question of detectability will be addressed in § III).

As an illustration, we compute the evolution of a SNR contained within a superbubble with radius $R \approx$ 105 pc. From the model calculations of Bruhweiler et 
al., such a shell would exist around a typical OB association after a few million years. The characteristics of the SNR within a superbubble are summarized in Table 1 at the end of each phase. We shall call these SNRs the hot cavity SNRs.

\section{c) A SN Surrounded by the Undisturbed ISM}

In some instances, an intermediate-mass star may become a SN outside molecular clouds and also outside the hot cavities surrounding $O B$ associations. The resulting SNR which interacts with the previously undisturbed interstellar medium of the galactic disk is the canonical SNR that has been described in $\S \mathrm{I}$. The characteristics of the canonical SNR are summarized in Table 1.

The canonical SNR has several intriguing differences in properties when compared to the other two types of SNRs. The total evolutionary lifetime is substantially longer than the lifetimes of either the molecular cloud SNR or the hot cavity SNR. The kinetics of a molecular cloud SNR are quickly transferred to the very massive molecular cloud, and the expanding shell stalls within $9 \times 10^{4}$ years for $n \sim 10^{4} \mathrm{~cm}^{-3}$. The hot cavity SNR, on the other hand, expands rapidly until it encounters the outer, slowly moving supershell of neutral gas (as observed by Heiles 1979). By then the SN shock is highly diluted, and consequently the ejecta are quickly decelerated. The slow accretion of material by the classical SNR in the undisturbed ISM extends the lifetime by more than twenty-fold compared to either alternate example.

\section{OBSERVABLE SNRS}

We discuss here the type of interstellar environment required to produce an observable SNR. By "observable" we mean a SNR which may be seen in $(a)$ visible light, (b) radio waves, or (c) X-rays.

\section{a) Visible Light SNRs}

The majority of visible light SNRs are believed to be in the Sedov (adiabatic) phase. Exceptions may be very "old" SNRs like the Monoceros Loop (although McKee and Cowie 1975 have suggested that even those are in the Sedov phase), or very "young" SNRs like $\mathrm{C}$ as $\mathrm{A}$ and the Crab Nebula. A SNR can be easily detected at visual wavelengths if the emission measure, $\mathrm{EM}=n_{e}^{2} L$, exceeds $50 \mathrm{~cm}^{-6} \mathrm{pc}$, where $n_{e}$ is the electron density in the SN shell and $L$ is the shell thickness. Careful observations will aid in detecting a SNR with $\mathrm{EM} \sim 20-50 \mathrm{~cm}^{-6}$ pc for $T \sim 10^{4} \mathrm{~K}$, but very special, tedious techniques (for example, a large Fabry-Perot etalon) are needed to detect a SNR with $\mathrm{EM} \sim 5 \mathrm{~cm}^{-6}$ pc.

The emission measure can be expressed as (assuming, roughly, an average path length of $R_{s} / 12$ in the
Sedov phase)

$$
\mathrm{EM}=\frac{4}{3} R_{s} n_{0}^{2}
$$

where $n_{0}$ is the ambient ISM density and $R_{S}$ is the radius of the shock front. Equation (7) is actually an overestimate because the density drops off rapidly behind the shock front (Spitzer 1978; Chevalier 1974).

The EM observability criterion (EM $\gtrsim 50 \mathrm{~cm}^{-6} \mathrm{pc}$ ) is a function of time. For the Sedov phase, we find

$$
n_{0} \gtrsim 1.8 E_{51}{ }^{-1 / 9} t_{4}-2 / 9 \mathrm{~cm}^{-3},
$$

where $\mathrm{EM} \gtrsim 50 \mathrm{~cm}^{-6}$ pc. Taking $E_{51}=1, t_{4} \sim 3$ (see Table 1C), we find that $n_{0} \gtrsim 1.4 \mathrm{~cm}^{-3}$ is required. Even for the SNR with $E M \gtrsim 5 \mathrm{~cm}^{-6} \mathrm{pc}$, we need $n_{0} \gtrsim 0.3$ $\mathrm{cm}^{-3}$.

For a SNR in the isothermal (cooling) phase, the observability estimates are different. Large compressions can take place in this case (Cox 1972; McCray, Stein, and Kafatos 1975). McCray, Stein, and Kafatos (1975) find that compression ratios as high as $\sim 70$ may be reached if the ambient magnetic field is weak or as low as $\sim 20$ when the magnetic field cannot be ignored ( $B>3 \times 10^{-6}$ gauss). The appropriate path length is the characteristic cooling length (McCray, Stein, and Kafatos 1975). For a $100 \mathrm{~km} \mathrm{~s}^{-1}$ shock with no ambient magnetic field (the most favorable case) the emission measure would exceed $50 \mathrm{~cm}^{-6} \mathrm{pc}$ if $n_{0} \gtrsim 0.8$ $\mathrm{cm}^{-3}$. Correspondingly, for $\mathrm{EM} \gtrsim 5 \mathrm{~cm}^{-6} \mathrm{pc}, n_{0} \gtrsim 0.08$ $\mathrm{cm}^{-3}$. Since the ambient densities are lower, it is more favorable to observe an optical SNR during its isothermal phase than during its adiabatic phase. For an "easy" detection, it seems that the shock must collide with a dense cloud (locally, the intercloud medium has a density of $\sim 0.15 \mathrm{~cm}^{-3}$; see Falgarone and Lequeux 1973).

The filling factor of clouds is quite small $(f \sim 1-10 \%$; see McCray and Snow 1979). Moreover, the number of clouds drops off rapidly with height above the galactic plane (the cloud scale height is probably roughly half of the scale height of the diffuse intercloud medium; see Falgarone and Lequeux 1973). Hence, few optically observable SNRs are expected at large heights above the galactic plane. The Cygnus Loop may be observable because it has collided with a neutral cloud or clouds. These clouds cannot be the same type of neutral clouds that produce $\mathrm{H}$ I absorption profiles, as the H I absorption clouds are found (Radhakrishnan et al. 1972) within $300 \mathrm{pc}$ of the galactic plane.

\section{b) Radio Observable SNRs}

Radio SNRs are much more numerous than either optical or X-ray remnants. About 130 radio SNRs have been observed in the Galaxy (Clark and Caswell 1976), whereas only 30 of the radio SNRs are detected by any 
optical emission (van den Bergh 1978). It is easier to detect a SNR at radio wavelengths both because lower ambient densities are necessary for radio detection and because dust does not absorb radio photons. It is usually assumed that van der Laan's (1962) theory applies to the older SNRs. However, the statistical investigation of Clark and Caswell (1976) confirmed that for the majority of SNRs the Sedov solution properly describes the value of the diameter $D$ with time. Clark and Caswell derived an average $\left\langle E / n_{0}\right\rangle \sim 5 \times$

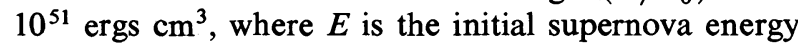
and $n_{0}$ is again the ambient density. If $E=10^{51} \mathrm{ergs}$, the averaged $n_{0}$ implied by their analysis is $0.2 \mathrm{~cm}^{-3}$. More recently, Caswell and Lerche (1979) refined the $\Sigma-D$ relation to include a $z$-dependence. $(\Sigma$ is the radio surface brightness.) The derived scale height of radio SNRs is $200 \mathrm{pc}$. The implied frequency of SN-producing radio SNRs within the Galaxy if $f \sim 1 / 80 \mathrm{yr}^{-1}$. The lower values of the ambient density implied by the radio observations confirm that SNRs are more easily detected in the radio.

There is also information on the galactic distribution of SNRs. Ilovaisky and Lequeux (1972) find that the distribution of radio SNRs closely follows the radial distribution of the nonthermal background radio emission. At $5 \mathrm{kpc}$ from the galactic center the radio SNRs are 3 times as abundant as at $10 \mathrm{kpc}$. Beyond $15 \mathrm{kpc}$ there are very few radio SNRs.

\section{c) X-Ray Observable SNRs}

Very few X-ray SNRs have been observed (for a recent review of the X-ray SNRs, see Clark and Culhane 1976), perhaps in part because of the limited sensitivity of complete X-ray surveys. The $E / n_{0}$ value derived for the X-ray SNRs support the average value obtained from radio observations (Gorenstein, Harnden, and Tucker 1974; Clark and Caswell 1976), although the two studies are vastly different. The thermal X-ray flux in the $1-100 \mathrm{keV}$ region of the spectrum for SNRs is given by Gorenstein, Harnden, and Tucker as $L_{X} \sim 5 \times$ $10^{33} n_{0}^{2} \mathrm{R}_{\mathrm{pc}}^{3}$ ergss ${ }^{-1}$. Using the above expression for $L_{X}$ and the X-rays measured for Pup A, the Cygnus Loop, and the Vela SNR, we conclude that an X-ray SNR is not observable if the ambient density is appreciably less than $\sim 0.1 \mathrm{~cm}^{-3}$. On the basis of recent $H E A O 2$ (Einstein) searches for X-ray emission of radio and optical SNRs (Knox Long, private communication), initial ambient densities below $0.05 \mathrm{~cm}^{-3}$ would not produce detectable X-ray fluxes. A value of the critical density of $0.1 \mathrm{~cm}^{-3}$ required to observe an X-ray remnant is consistent with the Einstein results. From the above discussion we conclude that if the ambient interstellar medium density $n_{0}$ exceeds a critical value $n_{c}$, the SNR is observable. It would most probably be observed as a radio SNR; but if $n_{0}$ is appreciably larger and the SNR is not too distant, it may be an optical or an X-ray observable SNR. We adopt the value $n_{c}=0.1$ $\mathrm{cm}^{-3}$ with the awareness that this is a realistic estimate really for the radio SNRs. In any case, we find that the study which follows would not change appreciably if $n_{c}$ were to change by a factor of 2 to 3 . For optical SNRs in the Sedov phase, we find $n_{c} \sim 1.0 \mathrm{~cm}^{-3}$, but this value is probably unimportant since it is much easier to detect the radio SNRs.

To simplify our analysis, we assume that all SNRs located within a medium with ambient density exceeding $n_{c}$ will be observable, but all SNRs located were $n_{0}<n_{c}$ are not observable. For example, SNRs with diameters approaching the cloud scale height would be expected to be brighter on their edge nearest to the plane (Clark and Stephenson 1977; Caswell and Lerche 1979) and therefore would be observable even though a portion of the SNR is located in a medium with density less than $n_{c}$. The largest observable SNRs have diameters $\lesssim 50$ pc which is less than the cloud scale height, so our results will not be significantly changed by this effect.

\section{STATISTICS OF SUPERNOVAE}

The average expansion rate for $\mathrm{OB}$ associations is $\sim 5 \mathrm{~km} \mathrm{~s}^{-1}$. Since the radius of a typical supercavity at the Sun's distance from the galactic center is $250 \mathrm{pc}$, it takes about $5 \times 10^{7}$ years for an association member to overtake the supershell. During this time all stars more massive than $7 M_{\odot}$ will have completed their evolution, so that if a lower mass limit of $8 M_{\odot}$ for stars that produce SNs is adopted, we find that very few stars are capable of escaping the cavity before they become supernovae (the numbers are higher for closer distances to the center-see below - but still small). If the mass limit of stars producing SNs is extended down to $4 M_{\odot}$, then the majority of SN explosions that produce SNRs in the solar neighborhood would originate in 4-8 $M_{\odot}$. A significant fraction of the progenitors would escape the low-density cavity before exploding. As there is an uncertainty of the mass limit of stars which produce SNs, we will consider two limits: $8 M_{\odot}$ and $4 M_{\odot}$ stars. In $\S$ IV $a$ below, we use $8 M_{\odot}$ while in $\S$ IV $b$ where we also include the effects of the gravitational field from the galactic disk, we adopt $4 M_{\odot}$ as the lower mass limit. By these two examples we represent the upper and lower limits to supernova occurrences within $O B$ associations.

Early type stars have a high incidence of binaries. A survey of early B stars (Abt and Levy 1978) shows that about half of these stars are in multiple systems.

Blaauw (1964) suggests that when a SN occurs in such a binary system, the companion can become a runaway star. In his classical work, 19 runaways are identified, out of which the latest spectral type is B3 with an assigned mass of $\sim 10 M_{\odot}$. A later study of 304 O stars (Cruz-Gonzalez et al. 1974) concludes that at 
least $20 \%$ of all $\mathrm{O}$ stars are runaway stars. Using the above evidence, and assuming that all binaries produce runaways, it follows that one-third of all SNs are from runaway progenitors. It is the runaway stars that have an opportunity to escape the superbubbles and in turn produce observable SNRs.

However, not all runaway progenitors produce observable SNRs. In order to estimate the fraction that produce observable SNRs, we need to estimate the following:

i) the initial mass function (IMF);

ii) the total evolutionary lifetime, $\tau$, for stars with different masses;

iii) the average peculiar velocity for runaway stars, $V_{p}$, which when used with the total evolutionary times will allow us to estimate the total distance $d$ that a runaway star would travel from the $\mathrm{OB}$ association;

iv) the effective critical scale height, $H_{c}$, for observable SNRs.

We now discuss each of these variables that will influence the estimate of observable SNRs.

The IMF for the massive stars is uncertain at best, especially for the $O$ stars. Ostriker, Richstone, and Thuan (1974) used the observational data of Richstone and Davidson (1972) to derive an IMF for O stars. Their mass function predicts significantly more massive $O$ stars than that predicted by an extrapolation of the IMF deduced for the mid to late B stars by McCuskey (1966). Theoretical evolutionary calculations, when compared with eclipsing binary data (Stothers 1972), indicate a different mass versus spectral type relationship from that used by Ostriker et al. Bruhweiler (1980) has reanalyzed the Richstone and Davidson (1972) data and has determined the masses for MK standards based upon the work of Stothers (1972). Both the data of Richstone and Davidson and of McCuskey (1966) can be represented by an IMF which relates the total number of stars as

$$
\phi \propto M^{-2.25}
$$

The total evolutionary time, $\tau$, is estimated using the mass versus total evolutionary time presented by Stothers (1972) for the case $X_{e}=0.739$ and $Z_{e}=0.021$.

We now estimate the distances $d$ that a runaway star travels from an $\mathrm{OB}$ association before it becomes a supernova:

$$
d=V_{p}\left(\tau_{2}-\tau_{1}\right)=V_{p} \Delta \tau_{21}
$$

where $\tau_{1}$ and $\tau_{2}$ are the total evolutionary times of the primary and the secondary in a binary system and $\Delta \tau_{21}$ is the difference. The average mass of the primary, $M_{1}$, can be expressed in terms of the mass of the secondary,
$M_{2}$, by the relation:

$$
\left(M_{1} / M_{2}\right)^{-2.25}=\frac{1}{2} .
$$

The higher mass limit for the IMF is not critical. In equation (11) we assume that the primary has a random mass distribution described by the IMF in equation (9). In Table 2, we present the resultant mass (which is a mean value) of the primary $M_{1}$, computed from equation (11), and the time scale $\Delta \tau_{21}=\tau_{2}-\tau_{1}$, which enters the expression in (10). ${ }^{4}$

The effective critical scale height, $H_{c}$, for observable SNRs is determined by the scale height of the gas. In Figure 1 we show the structure of the supercavity produced at three different distances from the galactic center, $R_{\mathrm{gal}}=5,10$, and $20 \mathrm{kpc}$. These are structures based upon the model we calculated (Bruhweiler et al. 1980). We assumed an exponential density distribution

$$
n=n_{0} e^{-z / H},
$$

with $H=70,150,500 \mathrm{pc}$ and $n_{0}=3,1,0.1 \mathrm{~cm}^{-3}$ for $R_{\mathrm{Gal}}=5,10,20 \mathrm{kpc}$, respectively. The assumed densities are appropriate for the $\mathrm{H}_{\mathrm{I}}$ medium as determined in Paul, Cassé, and Cesarsky (1976), and the scale height is from Kerr (1969). Exponential distributions were found by Celnik, Rohlfs, and Braunsfurth (1979) for large distances away from the galactic plane. They give

\footnotetext{
${ }^{4}$ In our discussion we have ignored the effects of mass loss and mass exchange in the evolution of $O$ and $B$ stars. These processes affect $\Delta \tau_{12}$, which in turn affects the fraction of runaway stars that escape from the supercavity.

Mass loss is expected to lengthen (by about $10 \%$ ) the evolutionary lifetimes of the more massive stars (Chiosi, Nasi, and Sreenivasan 1978). However, the domain of extensive mass loss is limited to B0 or earlier stars (Snow and Morton 1976), i.e., stars with initial masses $\geq 17 M_{\odot}$. From our calculations, these stars become supernovae either inside the supercavity or outside the galactic plane. Thus, the proposed increase in evolutionary time scales has a negligible effect.

A potentially more significant effect is due to the mass distribution of the binaries. For the sake of simplicity, we have assumed a random distribution of mass ratios among binaries. This assumption gives $M_{2} / M_{1} \geq 0.73$ for masses of interest in this work. On the other hand, if close binaries are formed as bifurcation products, then the mass ratio should be of order unity. Observationally determined values for this ratio range from 0.35 (Stone 1979) to about 1 (Heintze 1973). In view of these uncertainties, our assumption of $M_{2} / M_{1} \gtrsim 0.73$ is reasonable. If the true ratio were smaller, $\Delta \tau_{12}$ would be larger than our tabulated values, whereas the bifurcation hypothesis leads to smaller $\Delta \tau_{12}$ than our tabulated values. In any event, the effects are not overwhelming, and our procedure is justifiable at present. A final uncertainty is introduced by our having ignored the effect of mass transfer in computing $\Delta \tau_{12}$. Due to the speeding up of the evolution beyond core $\mathrm{H}$-exhaustion stage, the effects of mass dumping by the primary is expected to be minimal when compared to the shortening of the evolutionary time scale of the secondary. This would lead to generally smaller $\Delta \tau_{12}$. Since in order to produce a runaway the mass ejected must exceed the mass of the companion, mass transfer could modify the statistics of close binaries.
} 
TABLE 2

Primary and Secondary Masses of THE BINARY SYSTEM AND THE EVOLUTIONARY TIMe Scale Differences

\begin{tabular}{lcc}
\hline \hline$M_{1}\left(M_{\odot}\right)^{\mathrm{a}}$ & $M_{2}\left(M_{\odot}\right)^{\mathrm{b}}$ & $\Delta \tau_{21}(\mathrm{yr})^{\mathrm{c}}$ \\
\hline 25.85 & 19 & $2.8 \times 10^{6}$ \\
24.49 & 18 & $2.9 \times 10^{6}$ \\
23.13 & 17 & $3.7 \times 10^{6}$ \\
21.77 & 16 & $4.0 \times 10^{6}$ \\
20.4 & 15 & $4.2 \times 10^{6}$ \\
19.05 & 14 & $5.1 \times 10^{6}$ \\
17.69 & 13 & $5.4 \times 10^{6}$ \\
16.33 & 12 & $5.6 \times 10^{6}$ \\
15.0 & 11 & $7.9 \times 10^{6}$ \\
13.6 & 10 & $9.9 \times 10^{6}$ \\
12.24 & 9 & $1.49 \times 10^{7}$ \\
10.88 & 8 & $1.86 \times 10^{7}$ \\
9.53 & 7 & $2.43 \times 10^{7}$ \\
8.16 & 6 & $3.03 \times 10^{7}$ \\
6.8 & 5 & $4.25 \times 10^{7}$ \\
5.44 & 4 & $7.69 \times 10^{7}$ \\
\hline
\end{tabular}

${ }^{a}$ Average mass of the primary for the tabulated mass of the runaway secondary.

${ }^{b}$ Mass of the runaway secondary.

${ }^{c}$ Difference in the total evolutionary times, $\Delta \tau_{21}=\tau_{2}-\tau_{1}$, where $\tau_{1}$ is the evolutionary time of the primary and $\tau_{2}$ is the total evolutionary time of the runaway secondary. detailed formulae for the scale heights $H$, although the values of $n_{0}$ are harder to determine from their work. Assuming that $5 \%$ of the total mass of the galaxy is in gaseous form, we find-using the work of Celnik, Rohlfs, and Braunsfurth - that $n_{0} \sim 6,0.7$, and $4 \times 10^{-3}$ $\mathrm{cm}^{-3}$ for $R_{\mathrm{Gal}}=5,10$, and $20 \mathrm{kpc}$, respectively. Bohlin, Savage, and Drake (1978), on the other hand, find that $n_{0} \sim 0.9$ for the $\mathrm{H}$ I medium and $\sim 1.2$ for the $\mathrm{H} \mathrm{I}+\mathrm{H}_{2}$ medium (in the solar neighborhood).

Even though there are large uncertainties in the parameters of the ISM gas, the structure of the bubbleSN cavity is not affected very much. This is so because the radius of the bubble is only weakly proportional to the ambient density $\left(\propto n_{0}^{-1 / 5}\right)$ and the radius of the SN produced shell is proportional to $n_{0}^{-1 / 3}$ near the plane. For the case where the uncertainties are large $\left(R_{\mathrm{Gal}}=20\right.$ kpc) we find that even the higher density $n_{0}=0.1 \mathrm{~cm}^{-3}$ produces such large supercavities that essentially no runaway stars escape. Therefore, this result would still be true if we chose $n_{0}$ to be smaller.

\section{a) Supernova Statistics Derived with $8 M_{\odot}$ Lower Limit for SN Progenitors, and Ignoring Gravitational Effects of the Disk}

We now compute the percentage of all SN progenitors that produce observable SNRs. In this case we

$$
n=n_{0} e^{-z / H}
$$

$$
\begin{aligned}
& H=150 \mathrm{pc} \\
& n_{0}=1 \mathrm{~cm}^{-3}
\end{aligned}
$$

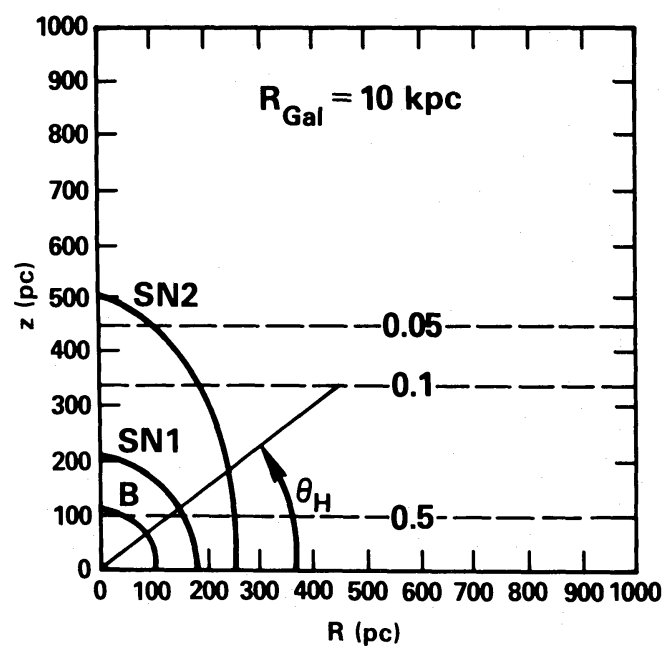

$$
\begin{aligned}
& H=70 \mathrm{pc} \\
& \mathrm{n}_{\mathrm{o}}=3 \mathrm{~cm}^{-3}
\end{aligned}
$$

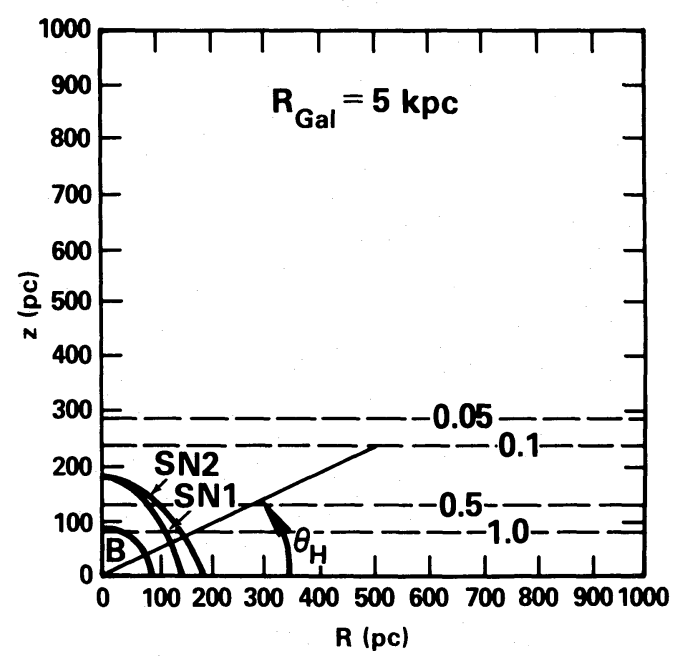

FIG. 1.-Geometry of the supercavity structures at $R_{\mathrm{Gal}}=5 \mathrm{kpc}$ and $10 \mathrm{kpc}$ as modeled by Bruhweiler et al. (1980). The three stages of evolution are depicted. The curve labeled $B$ is the limit of the supercavity formed by the 28 massive $O$ stars having significant stellar winds. The curve labeled SN1 defines the size of the shell at the evolutionary time when all 28 massive O stars have become SNs. However, 180 more B stars have masses greater than $8 M_{\odot}$. These too become SNs and drive the supercavity to the SN2 size. 
assume $8 M_{\odot}$ to be the lower limit for SN progenitors, and ignore the gravitational effects of the disk. The gravitational force of the disk tends to restore gas to the galactic plane and in the $z$-direction decreases the size of the supercavities. Hence, the percentages found in this case will be a lower limit to the actual percentage.

In Figure 2 we present the fraction of runaway stars in mass intervals $\Delta M=1 M_{\odot}$ that escape the supercavity to produce observable SNRs. The results change for different ratios of $H_{c} / V_{p}$; hence, we show the curves for a range of $H_{c} / V_{p}$. For example, if we assume $V_{p}=50 \mathrm{~km} \mathrm{~s}^{-1}$ and we want the appropriate curve for $R_{\mathrm{Gal}}=5 \mathrm{kpc}$, from Figure $1, H_{c}=240 \mathrm{pc}$ and therefore $H_{c} / V_{p}=4.8$. Similarly, for $R_{\mathrm{Gal}}=10 \mathrm{kpc}, H_{c}=350 \mathrm{pc}$ and $H_{c} / V_{p}=7$.

We now illustrate the application of this figure by presenting numerical estimates for the fraction of stars in a $1 M_{\odot}$ mass interval that escape the supercavity and produce an observable remnant. We chose $n=n_{0} e^{-z / H}$ as in Paper I at the two galactic distances $R_{\mathrm{Gal}}=5$ and $10 \mathrm{kpc}$. The critical density, $n_{c}=0.1 \mathrm{~cm}^{-3}$, makes the total fraction of supernovae producing observable remnants at $R_{\mathrm{Gal}}=20 \mathrm{kpc}$ equal to zero. Decreasing the critical density to $0.05 \mathrm{~cm}^{-3}$ would predict a total fraction at $R_{\mathrm{Gal}}=20 \mathrm{kpc}$ of less than $1 \%$.

Table 3 presents the lower limit on the percentage of SNs that produce observable SNRs. In column (1), rows $2-10$, we increment the mass range in bins of 1 $M_{\odot}$. However, row 1 has a bin of 17-70 $M_{\odot}$. In column (2), we present the fraction of all SN progenitors that are within the mass bin. In columns (3) and (4), the fraction of all SN progenitors, which are within the mass bin, is given for those that produce observable SNRs at 5 and $10 \mathrm{kpc}$. Column (5), which would list the fraction of $\mathrm{SN}$ progenitors that produce SNRs at $R_{\mathrm{Gal}}=20 \mathrm{kpc}$, is empty to emphasize that no $S N R s$ would be produced in the ambient interstellar gas at 20 $k p c$. In row 11 we add the incremental percentages to find the total percentage of runaway $\mathrm{SN}$ progenitors that produce observable SNRs. To this total, we must add in row 12 the few $\mathrm{SN}$ progenitors in the low mass range that survive long enough to escape the supercavity even at the association expansion velocity of $5 \mathrm{~km}$ $\mathrm{s}^{-1}$. By comparison, we have assumed that the runaway progenitors have $V_{p}=50 \mathrm{~km} \mathrm{~s}^{-1}$. We see that no slow moving stars escape the supercavity at $R_{\mathrm{Gal}}=10 \mathrm{kpc}$ while only $4.3 \%$ of the $\mathrm{SN}$ progenitors are slow moving stars which escape the supercavity of $R_{\mathrm{Gal}}=5 \mathrm{kpc}$.

Even if a SN explodes outside the supercavity formed by the parent association, such a $\mathrm{SN}$ might not be in the ambient interstellar gas. Rather, the $\mathrm{SN}$ may find itself in another supercavity and hence it would not form a visible SNR. The fraction of the ISM occupied by these supercavities is hard to estimate. The $O$ vi gas (McCray and Snow 1979) has a filling factor estimated to be $20 \%$ whereas the hot gas responsible for the X-ray

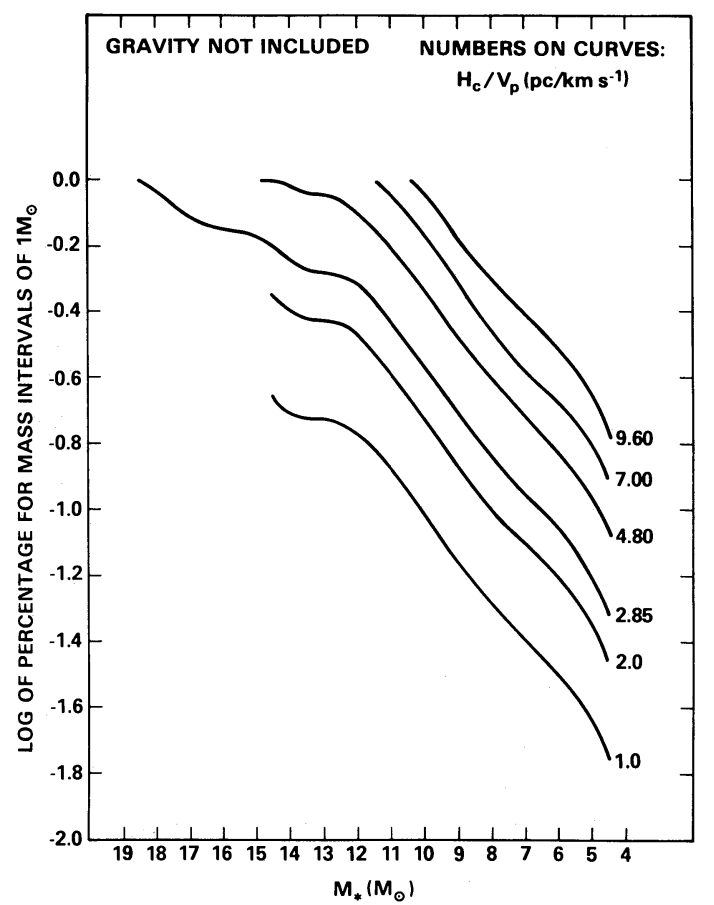

Fig. 2.-Fraction of runaway stars that escape the supercavity to produce observable SNRs. The various curves are for various assumptions of $H_{c} / V_{p}$ where $H_{c}$ is the critical $z$ distance beyond which a SNR would not be detectable and $V_{p}$ is the runaway star velocity. Note that here the gravitational restoring force is not considered for the runaway star.

background (Kraushaar 1977) has a filling factor estimated to be $50 \%$. We find that about $30 \%$ of the ISM is occupied by these superbubbles produced by OB associations. However, this is likely to be a lower limit since we do not include in our model the (older) B associations. With $30 \%$ of the ISM assumed to be in the hot phase, we find the percentages listed in the last row. Note that we have decreased the percentage of the runaway progenitors by one-third and not the percentage of the slow-moving progenitors, as the latter will be just beyond the superbubble and would be very unlikely within another supercavity.

\section{b) Supernova Statistics Derived with $4 M_{\odot}$ Lower Limit for the SN Progenitors, and Including Gravitational Effects of the Disk}

The runaway progenitors and the gas are subjected to a gravitational restoring force toward the disk which we have ignored thus far. Close to the galactic plane the gravitational force law can be approximated by $\ddot{z}=-k z$, with the resultant motion being that of an undamped harmonic oscillator.

We now calculate the critical angle, $\theta_{H}$, at which runaway progenitors would produce SNs at a height $H_{c}$ above the plane for a selected mass range as represented by $\Delta \tau_{21}=\tau_{2}-\tau_{1}$. In terms of the $z$-direction 
TABLE 3

A Lower Limtt on the Percentage of SNs That Produce Observable SNRs ${ }^{a}$

\begin{tabular}{|c|c|c|c|c|}
\hline \multirow{2}{*}{$\begin{array}{l}\text { SN PROGENITOR } \\
\text { MASS }\left(M_{\odot}\right) \\
\text { (1) }\end{array}$} & \multirow{2}{*}{$\begin{array}{l}\text { Relative Fraction } \\
\text { OF TOTAL SN } \\
\text { Progenitors } \\
(2)\end{array}$} & \multicolumn{3}{|c|}{$\begin{array}{c}\text { Percentage of SNs }{ }^{b} \\
\text { Producing OBSERVABLE SNRs }\end{array}$} \\
\hline & & $R_{\mathrm{Gal}}=5 \mathrm{kpc}$ & $R_{\mathrm{Gal}}=10 \mathrm{kpc}$ & $R_{\mathrm{Gal}}=20 \mathrm{kpc}$ \\
\hline 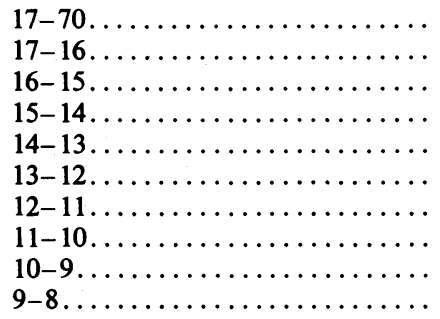 & $\begin{array}{r}17.9 \\
2.7 \\
3.3 \\
4.1 \\
5.2 \\
6.7 \\
8.7 \\
11.7 \\
16.3 \\
23.4\end{array}$ & $\begin{array}{l}0.0 \\
0.9 \\
1.1 \\
1.4 \\
1.6 \\
1.8 \\
2.0 \\
2.0 \\
2.1 \\
2.1\end{array}$ & $\begin{array}{l}0.0 \\
0.0 \\
0.0 \\
0.0 \\
0.4^{\mathrm{d}} \\
1.3 \\
2.4 \\
3.0 \\
3.2 \\
3.2\end{array}$ & $\begin{array}{l}\text { c } \\
\text { c } \\
\text { c } \\
\text { c } \\
\text { c } \\
\text { c } \\
\text { c }\end{array}$ \\
\hline $\begin{array}{l}\text { Runaway total } \ldots \ldots \ldots \ldots \ldots \\
\text { Additional contribution } \\
\text { by slowly moving stars. . . . . } \\
\text { e Total allowing for } 30 \% \text { ISM } \\
\text { as supercavities. . . . . . . . . }\end{array}$ & $100.0 \%$ & $4.3 \%$ & $0.0 \%$ & $\begin{array}{l}0.0 \% \\
0.0 \% \\
\end{array}$ \\
\hline
\end{tabular}

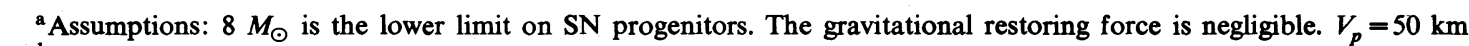
$s^{-1}$.

bंA density law $n_{0} e^{-z / H}$ is assumed with the following values: For $R_{\mathrm{Gal}}=5 \mathrm{kpc}, n_{0}=3 \mathrm{~cm}-3, H^{-3}=70 \mathrm{pc}$. For $R_{\mathrm{Gal}}=10$ kpc, $n_{0}=1 \mathrm{~cm}^{-3}, H=150 \mathrm{pc}$. For $R_{\mathrm{Gal}}=20 \mathrm{kpc}, n_{0}=0.1 \mathrm{~cm}^{-3}, H=500 \mathrm{pc}$.

c No observable SNRs will be produced at $20 \mathrm{kpc}$.

d The mass bin is only $13.0-13.5 M_{\odot}$.

' These total percentages are computed by assuming that one-third of the galactic plane is occupied by supercavities-i.e., it is computed by multiplying the runaway total by $2 / 3$ - but all slow moving stars contribute.
}

gravitational force, we can express $\dot{z}$ and $H_{c}$ :

$$
\dot{z}=V_{p} \sin \theta_{H}=A k^{1 / 2}
$$

and

$$
H_{c}=A \sin \left(k^{1 / 2} \Delta \tau_{21}\right),
$$

We can solve for $\theta_{H}$ as:

$$
\sin \theta_{H}=\frac{H \sqrt{k}}{V_{p}}\left[\sin \left(k^{1 / 2} \Delta \tau_{21}\right)\right]^{-1}
$$

Within the mass range represented by $\Delta \tau_{21}$, all runaway progenitors that are ejected from the galactic plane at ejection angles less than $\theta_{H}$ will produce SNs at heights less than $H_{c}$ above the galactic plane. The fraction of runaway progenitors that produce observable SNRs for a given mass range $\Delta M_{2}$ is then

$$
f_{\mathrm{SNR}}=\frac{2 \pi \int_{-\theta_{H}}^{\theta_{H}}\left[V_{p} \Delta \tau_{21}\left(\Delta M_{2}\right)\right]^{2} \cos \theta d \theta}{4 \pi\left[V_{p} \Delta \tau_{21}\left(\Delta M_{2}\right)\right]^{2}}=\sin \theta_{H}
$$

The fraction $f_{\text {SNR }}$ can be evaluated by substitution of equation (16) into equation (15).

We are aware that large uncertainties are inherent in the gravitational force law for the Galaxy. Although errors in the force could be quite large at high latitudinal distances, we are mostly concerned with $z$-distances less than $300 \mathrm{pc}$. The $\ddot{z}=-k z$ approximation has estimated errors of less than 20\% (see Paper I) in the solar neighborhood $\left(R_{\mathrm{Gal}} \approx 10 \mathrm{kpc}\right)$. However, at 5 and 20 $\mathrm{kpc}$ the force law is much more uncertain.

Based upon the curves published by Schmidt (1956), we adopted in Bruhweiler et al. (1980) linearized force laws out to $300 \mathrm{pc}$ in $z$ with the values of $k$ being $6.06 \times 10^{-15}, 2.58 \times 10^{-15}$, and $3.65 \times 10^{-16} \mathrm{~s}^{-2}$ for $R_{\mathrm{Gal}}$ $=5,10$, and $20 \mathrm{kpc}$, respectively.

Two other uncertainties are the values for $H_{c}$ and $V_{p}$. The derived values for $H_{c}$ depend upon the assumed values of $n_{0}$ and the assumed density law (eq. [12]). The average velocity, $V_{p}$, for runaway progenitors is also very uncertain. Several runaway stars are known to have $V_{p} \geq 100 \mathrm{~km} \mathrm{~s}^{-1}$ (Blaauw 1964; Stone 1979). The average runaway velocity we use is based on the velocities of 19 runaways presented by Blaauw. Various selection effects are known which strongly suggest the lower velocity runaways may not be recognizable as such. While a reasonable value for $V_{p}$ may be on the 


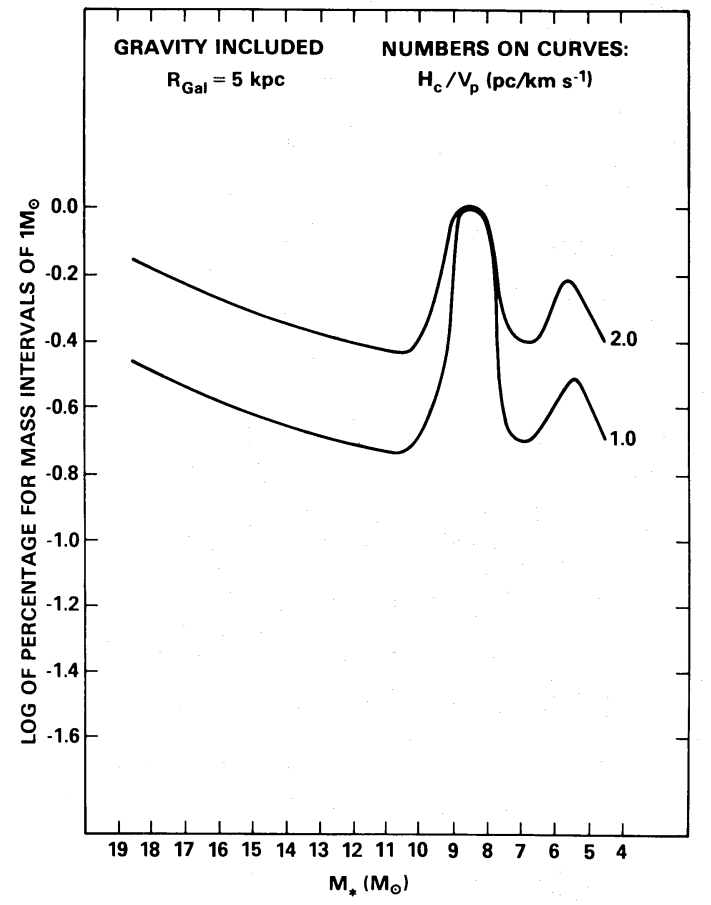

FIG. 3.-Fraction of runaway stars that escape the supercavity to produce observable SNRs. This is for $R_{\mathrm{Gal}}=5 \mathrm{kpc}$ and includes the gravitational restoring force.

order of $50 \mathrm{~km} \mathrm{~s}^{-1}$ (or lower), we feel constrained to express $H_{c}$ and $V_{p}$ in terms of one variable, namely, their ratio $H_{c} / V_{p}$.

We now show in Figures 3 and 4 curves similar to those in Figure 2, but with the gravitational force included. Within the mass intervals $\Delta M=1 M_{\odot}$, we plot the fraction of runaway progenitors that escape the supercavity and produce observable SNRs. Figure 3 is for $R_{\mathrm{Gal}}=5 \mathrm{kpc}$, and Figure 4 is for $R_{\mathrm{Gal}}=10 \mathrm{kpc}$. We present the results for the ratios of $H_{c} / V_{p}=1.0$ and 2.0.

The percentages of supernovae that would produce observable SNRs are summarized in Table 4. As in $\S \mathrm{IV} a$ we assume that one-third of all SNs are from runaway stars and that one-third of the interstellar medium is occupied by supercavities. The percentages listed in Table 4 are for an evolved supercavity with the internal SN from low-mass progenitors being at large distances from the shell (labeled in Fig. 1 as SN2). The SNR from the more massive stars have either dissipated or have been overtaken by the expanding supercavity around the old association. If stars appreciably less than $8 M_{\odot}$ produce $\mathrm{SNs}$, then it is possible that these older supercavities would be ringed with SNRs from the slow moving progenitors. For example, if the expansion velocity were $5 \mathrm{~km} \mathrm{~s}^{-1}$ for the association, then $5 M_{\odot}$ stars would diffuse $500 \mathrm{pc}$, which in the solar neighborhood is twice the shell radius, along the plane, by the time they become SNs.

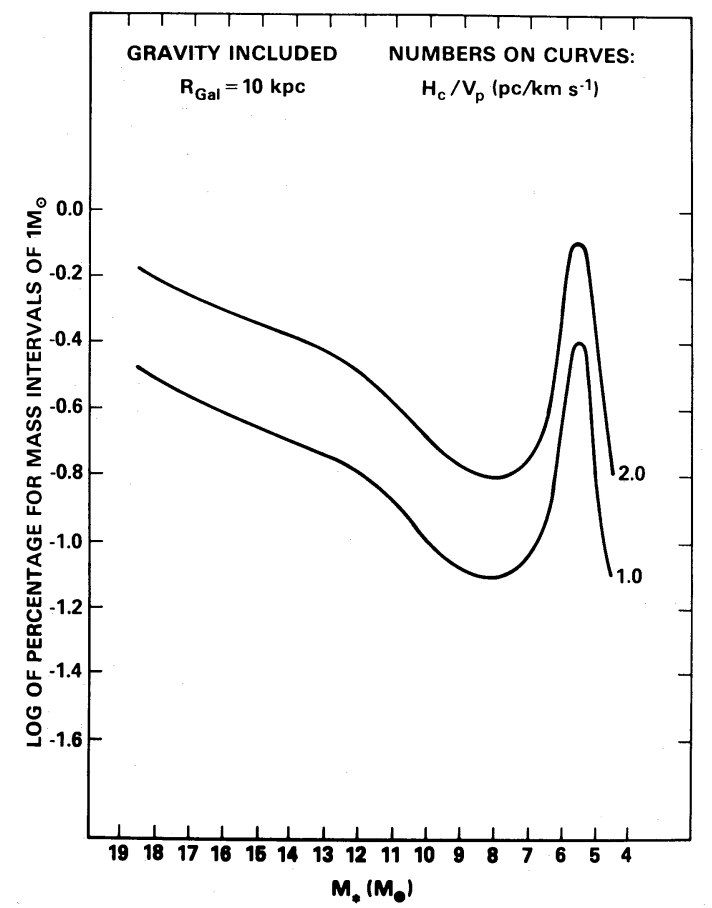

Fig. 4.- Fraction of runaway stars that escape the supercavity to produce observable SNRs. This is for $R_{\mathrm{Gal}}=10 \mathrm{kpc}$ including the gravitational restoring force

We have also computed similar models for young supercavities where only stars with spectral type B0 or earlier have become supernovae (the radius is shown in Fig. 1 as SN1). These younger supercavities would be surrounded by SNRs from the relatively more massive progenitors $\left(M \geq 11 M_{\odot}\right)$. A possible example of such a system may be the Gum Nebula. It is a roughly spherical cavity with a $125 \mathrm{pc}$ radius. Two known SNRs in close proximity are Vela X-1 and Puppis A.

The computed estimates of supernovae producing SNRs for the relative younger and older supercavities are very similar. The total percentages, summarized in Table 4 , are $29.8 \%$ and $23.1 \%$ for $R_{\mathrm{Gal}}=5 \mathrm{kpc}$ and 10 $\mathrm{kpc}$, respectively. About one-half of the SNRs are from the slow moving $\left(5 \mathrm{~km} \mathrm{~s}^{-1}\right)$ stars.

We also computed the percentages of SNs creating observable SNRs at $R_{\mathrm{Gal}}=20 \mathrm{kpc}$ and find that less than $1 \%$ of the supernovae would yield observable SNRs.

\section{DISCUSSION AND CONCLUSIONS}

Several important results are derivable from Tables 3 and 4.

The presence of supercavities drastically changes the mass distribution of $\mathrm{SN}$ progenitors that produce the classically detectable SNRs.

A greater percentage of higher mass stars escape the supercavity at small $R_{\mathrm{Gal}}$, primarily because the sizes 
TABLE 4

\begin{tabular}{|c|c|c|}
\hline \multirow{2}{*}{$\begin{array}{l}\text { MASS BIN } \\
\left(M_{\odot}\right)\end{array}$} & \multicolumn{2}{|c|}{$\begin{array}{c}\text { Percentage of SN } \\
\text { Producing Observable SNR }\end{array}$} \\
\hline & $R_{\mathrm{Gal}}=5 \mathrm{kpc}$ & $R_{\mathrm{Gal}}=10 \mathrm{kpc}$ \\
\hline 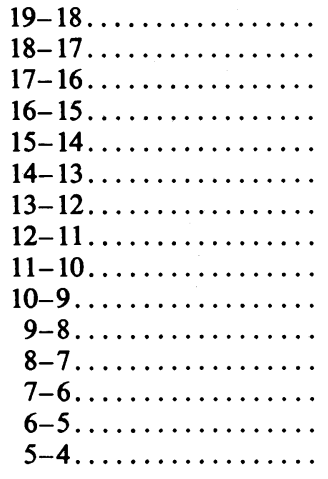 & $\begin{array}{l}0 \\
0 \\
0.12 \\
0.23 \\
0.29 \\
0.36 \\
0.46 \\
0.61 \\
0.72 \\
1.13 \\
1.63 \\
2.45 \\
3.85 \\
6.8 \\
12.39\end{array}$ & $\begin{array}{l}0 \\
0 \\
0 \\
0 \\
0 \\
0.36 \\
0.46 \\
0.61 \\
0.67 \\
0.72 \\
0.89 \\
1.35 \\
2.67 \\
6.8 \\
7.22\end{array}$ \\
\hline $\begin{array}{l}\text { Runaway total } \ldots \ldots \ldots \\
\text { Slowly moving stars ... } \\
\text { Total }{ }^{b} \ldots \ldots \ldots \ldots \ldots\end{array}$ & $\begin{array}{r}31.05 \% \\
9.13 \% \\
29.8 \%\end{array}$ & $\begin{array}{l}21.75 \\
8.6 \% \\
23.10 \%\end{array}$ \\
\hline
\end{tabular}

a The same density law as in Table 3 is assumed.

${ }^{b}$ This total is obtained by multiplying row 16 by $\frac{2}{3}$ (i.e., we assume as before that one-third of all ISM is occupied by cavities) and adding it to row 17.

of the supercavities are smaller toward the galactic center. The model prediction that more supernovae produce detectable SNRs at small $R_{\mathrm{Gal}}$ is in qualitative agreement with the observed distribution (van den Bergh 1978; Clark and Caswell 1976; Ilovaisky and Lequeux 1972). Had we chosen $n_{0}$ to be $6 \mathrm{~cm}^{-3}$ - which may be more appropriate for the dense inner arms-we would have predicted 2.2 times more SNRs at $5 \mathrm{kpc}$ than at $10 \mathrm{kpc}$. Due to the uncertainties involved, we consider this to be satisfactory agreement with the observations. This also implies that our assumption that the rate of SN outbursts throughout the galactic plane is nearly uniform may be close to reality. The distribution of observable SNRs is determined only by the presence and size of the supercavities and by the fraction of "runaway" SN progenitors that escape before becoming SNs.

The exact fraction of SNs that produce observable SNRs cannot be estimated accurately with the present data. Upon a variety of assumptions and parameters hopefully encompassing the real situations, we find that this fraction ranges from approximately $10 \%$ to $35 \%$, depending on the $R_{\mathrm{Gal}}$, with $5 \%$ variations in either direction being reasonable.

The interstellar gas densities affect the above percentages somewhat. However, the fraction of SNs producing observable SNRs more than doubles when the lower mass cutoff decreases from $8 M_{\odot}$ to $4 M_{\odot}$. Moreover, if $4 M_{\odot}$ stars are progenitors, the slowly moving stars which diffuse at the association velocity of expansion $\left(5 \mathrm{~km} \mathrm{~s}^{-1}\right)$ would make an equal contribution to the total SNR percentage.
Since our model indicates that only one to three out of every 10 supernovae produce an observable SNR, the disagreement between rates deduced from observable SNRs on the one hand and SNs and pulsars on the other hand can be understood. Tammann (1974) suggests a mean interval between $\mathrm{SNs}, \tau_{\mathrm{SN}} \sim 30$ years. This leads to a SNR production rate of one every $100-300$ yr. On the other hand, pulsar statistics (Taylor and Manchester 1977) imply $\tau_{\mathrm{SN}} \sim 10$ years. This leads to a SNR production rate of one every $30-100$ years. Since the mean $\tau_{\mathrm{SNR}}$ derived by Caswell and Lerche (1979) is $\sim 80$ years, there is a weak support for the higher SN rate. However, regardless of whether a SN occurs once every 10 or 30 years, we can now understand how both these numbers are lower than the 80 year interval for the production of detectable SNRs derived from radio data.

Recently, Higdon and Lingenfelter (1980) have proposed an alternative to our point of view. They propose that if a hot $\left(\sim 10^{6} \mathrm{~K}\right)$, tenuous gas fills $90 \%$ of interstellar space, the observed number and surface brightness distribution of galactic remnants implies a SN rate of one every $\sim 30 \mathrm{yr}$. However, even though in this way the statistics may equally be reconciled, we feel that their filling factor is excessively large, although not inconsistent with the $\mathrm{O}$ VI data (Jenkins and Meloy 1974). Our mechanism would operate in any case, further reducing the number of observable SNRs. Because of this, the $90 \%$ filling factor is not justified. The results of Tables 3 and 4 would indicate that filling factors as large as $70 \%$ could be tolerated, but not appreciably higher than that.

We must emphasize that our discussion has been confined to Type II supernovae. Type I supernovae, which probably originate from low-mass Population II stars, share almost none of the considerations addressed in this paper, and they should be investigated seperately. However, since some statistical studies of SNs do not differentiate the SN types, they cannot be compared with our results without previously estimating the Type I SN contribution. If one, however, examines the known SNs of the last millenium, all of which left observable remnants, the question of differentiating between SNs of Type I and SNs of Type II becomes important.

We conclude that the superbubble cavity conceptthat of a hot, low-density gas around stellar associations created by stellar winds and $\mathrm{SNs}$ - provides a most effective scenario for understanding the general structure of the interstellar medium. Moreover, we believe that supercavities have already been detected observationally as we (Bruhweiler et al. 1980) have pointed out previously for the H I supershells (Heiles 1979). More recently, aspects of our model have been adopted by Cash et al. (1980) to explain the X-ray superbubble in Cygnus. This provides a compelling argument that many supernovae do not occur in environments conducive to detectable SNRs. 


\section{REFERENCES}

Abt, H. A., and Levy, S. G. 1978, Ap. J. Suppl., 36, 241.

Blaauw, A. 1964, Ann. Rev. Astr. Ap., 2, 213.

Bohlin, R. C., Savage, B. D., and Drake, J. F. 1978, Ap. J. 224, 132.

Bruhweiler, F. C. 1980, in preparation.

Bruhweiler, F. C., Gull, T. R., Kafatos, M., and Sofia, S. 1980, Ap. J. (Letters), 238, L27.

Burton, W. B. 1976, Ann. Rev. Astr. Ap., 14, 275

Cash, W., Charles, P., Bowyer, S., Walter, F., Garmire, G., and Riegler, G. 1980, Ap. J. (Letters), 238, L71.

Caswell, J. L., and Lerche, I. 1979, M.N.R.A.S., 187, 201.

Celnik, W., Rohlfs, K., and Braunsfurth, E. 1979, Astr. Ap., 76, 24.

Chevalier, R. A. 1974, Ap. J., 188, 501. 1975, Ap.J., 198, 355. 1977, Ann. Rev. Astr. Ap., 15, 175.

Chevalier, R. A., and Theys, J. C. 1975, Ap. J., 195, 53.

Chiosi, C., Nasi, E., and Sreenivasan, S. R. 1978, Astr. Ap., 63, 103.

Clark, D. H., and Caswell, J. L. 1976, M.N.R.A.S., 174, 267

Clark, D. H., and Culhane, J. L. 1976, M.N.R.A.S., 175, 573.

Clark, D. H., and Stephenson, F. R. 1977, The Historical Supernovae (Oxford: Pergamon Press).

Cox, D. P. 1972, Ap. J., 178, 143.

Cruz-Gonzalez, C., Recillas-Cruz, E., Costero, R., Peimbert, M., and Torrés-Peimbert, S. 1974, Rev. Mexicana Astr. Ap., 1, 211. Elmegreen, B. G. 1979, preprint.

Falgarone, E., and Lequeux, J. 1973, Astr. Ap., 35, 253.

Gorenstein, P., Harnden, F. R., and Tucker, W. H. 1974, Ap. J., 192, 661 .

Gorenstein, P., and Tucker, W. H. 1976, Ann Rev. Astr. Ap., 14, 373.

Heiles, C. 1979, Ap. J., 229, 533.

Heintze, J. R. W. 1973, in IAU Symposium 54, Problems of Calibration of Absolute Magnitudes and Temperatures of Stars, ed. B. Hauck and B. E. Westerlund (Dordrecht: Reidel), p. 231

Higdon, J. C., and Lingenfelter, R. E. 1980, preprint.
Ilovaisky, S. A., and Lequeux, J. 1972, Astr. Ap., 18, 169.

Jenkins, E. B., and Meloy, D. A. 1974, Ap. J. (Letters), 193, L121.

Kerr, F. J. 1969, Ann. Rev. Astr. Ap., 7, 39.

Kraushaar, W. L. 1977, Review Lecture, 149th meeting, AAS.

Landau, L. D., and Lifshitz, E. M. 1959, Fluid Mechanics (Oxford: Pergamon Press).

Mansfield, V. N., and Salpeter, E. E. 1974, Ap. J., 190, 305.

McCray, R., and Snow, T. P. 1979, Ann. Rev. Astr. Ap., 17, 213.

McCray, R., Stein, R. F., and Kafatos, M. 1975, Ap.J., 196, 565.

McCuskey, S. W. 1966, Vistas in Astronomy, 7, 141.

McKee, C. F., and Cowie, L. L. 1975, Ap. J., 195, 715.

McKee, C. F., and Ostriker, J. P. 1977, Ap. J., 218, 148

Ostriker, J. P., Richstone, D. D., and Thuan, T. X. 1974, Ap. J. (Letters), 188, 187.

Paul, J., Cassé, M., and Cesarsky, C. J. 1976, Ap. J., 207, 62.

Radhakrishnan, V., Murray, J. D., Lockhart, Peggy, and Whittle, P. J. 1972, Ap. J. Suppl., 24, 15.

Richstone, D. O., and Davidson, K. 1972, A.J., 77, 298.

Schmidt, M. 1956, Bull. Astr. Inst. Netherlands, 13, 15.

Sgro, A. 1975, Ap. J., 197, 621

Shull, J. M. 1980, $A p$. J., $237,769$.

Silk, J., and Burke, J. R. 1974, Ap. J., 190, 11

Snow, T. P. 1979, preprint.

Snow, T. P., and Morton, D. C. 1976, Ap. J. Suppl., 39, 429.

Spitzer, L. 1978, Physical Processes in the Interstellar Medium (New York: Wiley)

Stone, R. C. 1979, Ap. J., 232, 520.

Stothers, R. 1972, Ap. J., 175, 431.

Tammann, G. A. 1974, in Supernovae and Supernova Remnants, ed. C. B. Cosmovici (Dordrecht: Reidel), p. 155.

Taylor, J. H., and Manchester, R. N. 1977, Ap. J., 215, 885.

van den Bergh, S. 1978, Ap. J. Suppl., 38, 119.

van der Laan, H. 1962, M.N.R.A.S., 124, 179.

Wheeler, J. C., Mazurek, T. J., and Sivaramakrishnan, A. 1980, Ap. J., 237, 781.

Woodward, P. R. 1976, Ap. J., 207, 484.

Frederick C. Bruhweiler: Computer Sciences Co., IUE Observatory, Code 685, NASA Goddard Space Flight Center, Greenbelt, MD 20771

Theodore R. Gull: Code 683, NASA Goddard Space Flight Center, Greenbelt, MD 20771

Minas Kafatos: Department of Physics, George Mason University, Fairfax, VA 22030

SABatino Sofia: Code 961, NASA Goddard Space Flight Center, Greenbelt, MD 20771 